\title{
Impact of spouse caregiving on health behaviors and physical and mental health status
}

\author{
Cathleen M Connell, PhD
}

\begin{abstract}
The impact of caring for a spouse with a progressive dementia on caregiver's health behaviors and health status was examined. Data collected from 44 spouse caregivers indicates that:

- Providing full-time care interferes with preventive health behaviors (eating nutritiously, exercising) and contributes to high risk behaviors (overeating, alcohol and substance use);

- Health behaviors are frequently used as coping strategies;

- Caregivers rated their own health as poorer than their spouse's health; and

- Disabling (arthritis, cardiac and back problems) and stress-related health problems (migraines, colitis) are a consequence of and interfere with care provision.
\end{abstract}

Cathleen M Connell, PhD, is Assistant Professor, Department of Health Behavior and Health Education, School of Public Health, and Director, Education and Information Transfer Core, Michigan Alzheimer's Disease Research Center, University of Michigan, Ann Arbor, Michigan.

\section{Introduction}

Increasing numbers of older adults are providing care to a spouse with a chronic illness. As reported in a national profile of caregivers of older adults, over one-third of all caregivers are spouses and almost three fourths are over the age of $65 .^{1}$ Spouses provide the most comprehensive care, are typically responsible for the most disabled individuals, and maintain the role of caregiver for a longer period than other caregivers. ${ }^{2}$ At the same time, spouses are the most vulnerable caregivers due to their own advancing age and concomitant increased prevalence of chronic illness and potentially diminished physical capacity. ${ }^{3,4}$ The health impact of caregiving is particularly salient among adults caring for a spouse because the continued provision of in-home care may be dependent on the successful management of the caregiver's physical health.

The correlates and mental health consequences of caregiving, including depression, lowered morale and well-being, emotional exhaustion, anxiety, helplessness, and interference with social and recreational pursuits have been well documented. ${ }^{5-8}$ The consequences of providing fulltime care to a family member for the caregiver's physical health have also received attention. ${ }^{4,9-17}$

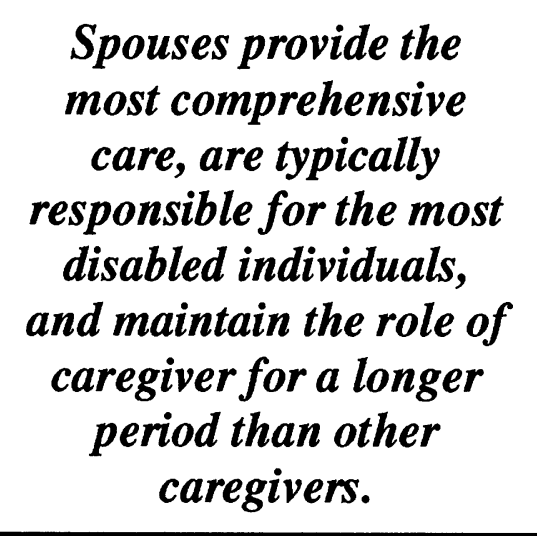

The majority of studies in which the physical health effects of caregiving have been examined have assessed self reports of physical health status and/or illness-related symptoms. ${ }^{4}(1)$ Caregivers perceive themselves to be in poorer health than their age peers, ${ }^{1}$ and this decline is positively correlated with the length of time in the caregiving role. ${ }^{9}$ Caregivers report that their health status has declined since providing care to a family member, ${ }^{9,18}$ especially among husbands caring for a wife with dementia ${ }^{17}$ and that health status is negatively related to the perceived burden of care provision. ${ }^{18}$ Caregiver's physical health concerns (i.e., fractures from falls, 
heart attacks, ulcers, and exhaustion $^{8}$ and psychotropic drug use ${ }^{19}$ were frequently cited as reasons for institutionalizing the patient for whom they were providing care.

Additionally, caregivers reported higher rates of diabetes, arthritis, ulcers, anemia, ${ }^{10,20}$ hypertension, and heart attacks ${ }^{21}$ than comparable population-based samples. A variety of health problems have been associated with demands of providing full-time care to a spouse, including cardiac, stomach and back problems, sleep disturbances, weight changes, peptic ulcers, alcohol and substance abuse, 9,22 and hypertension. ${ }^{17}$ Compared to other family caregivers, spouses reported the most health problems (i.e., more doctor visits, lower health self ratings, and more psychotropic drug use) and lower mental health ratings (i.e., more stress symptoms, lower ratio of positive to negative affect, and lower life satisfaction). ${ }^{3}$ Health problems related to caregiving, physical difficulties with care related tasks (transferring, lifting), being unable to engage in self-care, and being hospitalized as a result of an illness that began since providing care was reported among spouse caregivers. ${ }^{23}$

Physical health consequences may be especially acute for caregivers who live with and provide care for a spouse with dementia due to the chronic and unique stress associated with the role. ${ }^{3,17,24,25}$ Demented patients progressively lose both their ability to perform activities of daily living and their capacity for intimacy and companionship, exhibit cognitive and behavioral declines, may wander or exhibit sleep disturbances ${ }^{2,11}$ and may eventually lose their personality ${ }^{10}$ and sense of self. Caregivers' responses to these changes may be disruptive to patterns of daily self-care and physically and emotionally exhausting and destructive.

\section{The health impact of caregiving is particularly salient among adults caring for a spouse ...}

The present study was conducted to contribute to this literature on the physical health effects of caregiving by including a focus on the impact of providing care to a spouse with dementia on the caregiver's health behavior. The relationship between health behaviors (including physical activity, cigarette smoking, alcohol consumption, sleep patterns, and maintaining ideal body weight) and overall health status and morbidity have been well documented (see, for example, ${ }^{26-29}$. For older adults, many of whom have at least one chronic illness, health behaviors also play an important role in the self-management of existing conditions and in the prevention of future health problems or illness complications. Although the self-help literature ${ }^{21,24}$ has emphasized the need for caregivers to attend to their own health, limited attention has been paid to health behaviors in the empirical literature. The emotional, behavioral, and logistic demands of providing full-time care may interfere with preventive health behavior (exercise, eating nutritiously) and trigger high risk health behaviors (smoking, drug use). Relevant research that addresses this issue is not conclusive. For example, alcohol and drug use, eating, and smoking were viewed as ways to cope with the stress of caregiving in one study, ${ }^{16}$ but these behaviors were unrelated to the cognitive functioning of an ill spouse in another recent study of caregivers. ${ }^{17}$

The goals of this exploratory study include:

- To assess caregiver's health behavior and health status change since providing care to their spouse with dementia;

- To assess coping strategies with caregiving stress that involve health behaviors;

- To compare caregiver's selfratings of their own and their spouse's health status; and

- To examine relationships among subjective and objective caregiver burden and physical and mental health outcomes.

This study represents the baseline phase of a short-term longitudinal study based on daily health diaries completed by the present sample of dementia caregivers. ${ }^{30,31}$ An understanding of the health consequences of caregiving is essential to inform interventions designed to preserve the health of caregivers and their ability to perform caregiving tasks. In addition, because of the reciprocal relationship between physical and mental health, ${ }^{13,32-34}$ such interventions may ultimately prevent or lessen the potential mental health consequences (i.e., depression) of the caregiving role. ${ }^{10,12,20}$

\section{Procedure and sample}

A letter explaining the purpose of the research was sent to all caregivers of patients who were treated 
at a cognitive disorders clinic of a university medical school $(\mathrm{N}=89)$. Individuals were asked to return a self-addressed stamped post card indicating whether they would be willing to be involved in the study. Of the 69 individuals who returned a post card, 51 completed a mailed questionnaire (the majority of the 18 individuals who indicated that they were not willing to complete a questionnaire noted that a change in their caregiving status precluded their involvement in the study). Data from seven of the 51 respondents was excluded, resulting in a final sample of 44 individuals (data provided by an adult daughter, a caregiver whose spouse died before the questionnaire was received, and five caregivers of nursing home residents was deleted).

The 44 spouse caregivers in the final sample included 23 males and 21 females (see Table 1). Mean age was 68.2 years $(\mathrm{SD}=9.1$, range $=$ 43-84). The average educational level was 14.1 years $(S D=3.4$, range =8-22). All participants were white and resided with their spouse. Most caregivers (70 percent) were providing care to a spouse who had been diagnosed by a physician (most commonly by a neurologist) with Alzheimer's disease $(n=31)$; other diagnoses included Progressive $\mathrm{Su}$ pra Nuclear Palsy $(n=6)$, Parkinson's disease $(n=2)$, Pick's disease $(\mathrm{n}=1)$, and an unspecified dementia $(n=1)$. The spouse caregivers had been providing care for an average of 3.3 years $(\mathrm{SD}=2.3)$. Caregivers reported being diagnosed with an average of two chronic illnesses or conditions $(\overline{\mathrm{X}}=2.2, \mathrm{SD}=0.7)$; arthritis, hypertension, heart disease, atherosclerosis, cataracts, and diabetes were most prevalent (reported by $38.6,34.1,22.7,18.2$, and 11.4 percent of the sample, respectively). Mean depression (CES-D) score was 19.8 ( $\mathrm{SD}=11.9)$. As discussed by Biegel et al., ${ }^{25}$ CES-D scores range from 5.6 to 28.9 in previous research completed with samples of caregivers, with a modal score of 17. Older adults residing in the community typically score between 8 and 9 , and CES-D scores above 16 indicate being at risk for clinical depression. Thus, the majority of research (including the present study) indicates elevated rates of depressive symptoms among caregivers. ${ }^{25}$

\section{Measures}

A mailed questionnaire, designed to address the unique purpose of the present study and to be comparable to previous caregiving research, includes the following sections:

- Demographics;

- Caregiving history;

- Patient's behavior and care needs;

- Caregiver's physical health status, symptomatology, depression, and health behaviors.

Specific items, scales, and open- ended questions, organized by the four specific aims of the study, are described next.

\section{Caregiver's health behavior and health status change}

Five one-item indicators were included to determine changes in health behaviors since providing care to a spouse. For example, participants were asked: "Compared to the time before you began caring for your spouse, has the frequency with which you eat balanced, nutritious meals decreased, stayed the same, or increased?"

This question format was also used to assess changes in appetite, exercise, smoking, and alcohol intake since providing care to their spouse. Respondents were also asked: "In the past month, have you taken any medicine or anything else (e.g., alcoholic beverages) to help you sleep, calm you down, or perk you up?"

The physical health impact of caregiving was assessed by the question "has your physical health been affected by caring for your spouse?" For respondents who answered "yes," space was provided to

Table 1. Demographic characteristics of spouse caregivers.

$$
\begin{aligned}
& \text { Age } \\
& \text { Education (years) } \\
& \text { Caregiving (years) }
\end{aligned}
$$

Male

Female

Spouse's diagnosis

Alzheimer's

Supra Nuclear Palsy

Parkinson's disease

Pick's disease

\begin{tabular}{rrr}
\multicolumn{1}{c}{$\overline{\mathbf{X}}$} & SD & Range \\
\hline 68.2 & 9.1 & $43-88$ \\
14.1 & 3.4 & $8-22$ \\
3.3 & 2.3 & $0-12$
\end{tabular}

\begin{tabular}{cc}
$\mathbf{N}$ & $\%$ \\
\hline 23 & 52.3
\end{tabular}

21

47.7

$\begin{array}{rr}31 & 70.5 \\ 6 & 13.6 \\ 2 & 4.5 \\ 1 & 2.3\end{array}$


respond to a second question, "If so, in what way?" Physical health limitations were assessed by the question: "How much do health problems stand in the way of your doing the things you want to do?" Response choices include: a great deal, a little, or not at all. For respondents who indicated that health problems did interfere with their activities, space was provided to record an open-ended response to "If so, in what way?"

\section{Health behavior coping strategies}

Six items were included to assess the extent to which caregivers reported increasing the frequency with which they engaged in specific health behaviors during the past months as a way to cope with the stress of caregiving. The items included:

- "Comforted myself with food,"

- "Drank alcohol,"

- "Exercised,"

- "Took medications," and

- "Spent more time sleeping."

Response choices included: never, rarely/seldom, sometimes, often and most of the time.

\section{Comparison of caregivers and spouse's physical health}

A one-item indicator, "How would you rate your overall physical health at the present time?" was included. Response choices, arranged on a 4-point scale, included: poor, fair, good, and excellent. This item has been used extensively with community samples ${ }^{35}$ and caregivers. $^{17,20}$ Self-rated physical health has been shown to be highly related to objective measures of health and predictive of mortality. ${ }^{36,37}$ Using the same format, respondents were also asked, "How would you rate

\section{Forty percent of the respondents reported that their physical health had been negatively affected by caring for their spouse.}

your spouse's overall physical health at the present time?" ${ }^{20}$ Caregivers were asked if they have been diagnosed with any of 21 chronic illnesses and conditions.(2) A summary score was created by adding the total number of chronic illnesses or conditions reported. Scores could range from 0 to 21 . Caregivers were asked if their spouse was diagnosed with any of the same 21 chronic illnesses and conditions, and a comparable summary score was created.

Relationships among perceived and objective caregiver burden and physical and mental health variables

Perceived caregiver burden was assessed by a single item, "Overall, how burdened do you feel in caring for your spouse?" Response choices included: not at all burdened, a little burdened, moderately burdened, greatly burdened, and very greatly burdened. As discussed by Pruchno et al., ${ }^{13}$ this single item correlates highly with a composite-item burden index. ${ }^{38}$ Objective burden was assessed by two one-item indicators. The amount of time caregivers spend providing daily care was determined by the item: "About how many hours a day in the past month have you felt on duty in taking care of your spouse?" The actual number of hours, on-duty hours, was recorded. To assess the length of time spent in the caregiving role, caregiving years, a single item was included: "When did you start doing things for your spouse that he/she used to do for him/herself?" Selfcare was assessed by the item: "In general, how well would you say that you take care of yourself?" Response choices on a 3-point scale included: "Very well," "Fairly well," or "I neglect my own health."

The number of sick days was assessed by the item: "During the past six months, about how many days were you so sick that you were unable to carry on your usual activities such as working around the house or going to work? The number of days indicated by the respondent was recorded. The 12-item somatization subscale of the Hopkins Symptom Checklist (HSCL; 58 items) was included. ${ }^{39}$ Respondents indicated the frequency with which they experienced complaints with:

- Cardiovascular, gastrointestinal, and respiratory systems;

- Headaches, muscle pain, and discomfort; and

- Somatic symptoms associated with anxiety during the past week.

Response choices included: rarely/none, sometimes, occasionally, and most of the time.

Depression was assessed with the 20-item Center for Epidemiological Studies Depression Scale (CES-D), a highly reliable measure used extensively with community ${ }^{40}$ and 


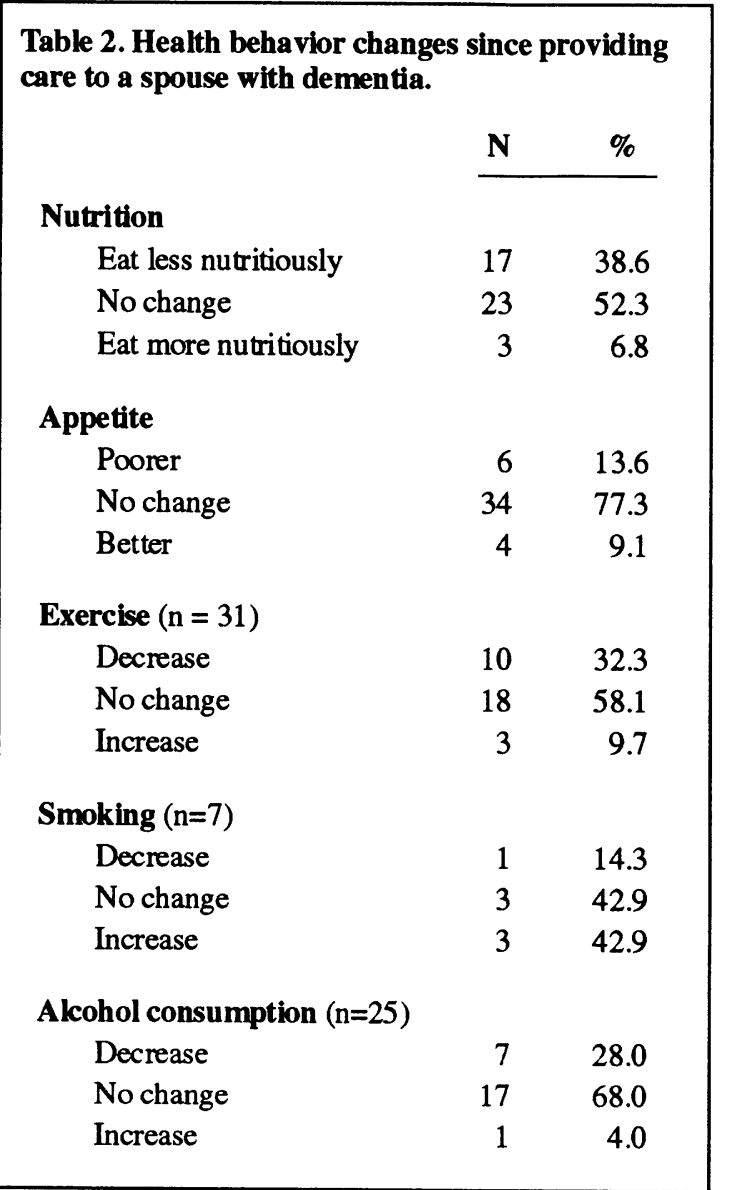

caregiver samples. ${ }^{12,17,20}$ Elevated CES-D scores (16 or higher) reflect symptoms that may accompany clinical depression. ${ }^{40,41}$

Worry was assessed by the item: "How often would you say you worry about things?" Response choices included: hardly ever, fairly often, often, and very often. Spirits was assessed by the item: "In general, would you say that most of the time you are in very low spirits, or very good spirits?"

\section{Results}

\section{Caregiver's health behavior and health status change}

Over one-third of the caregivers (39 percent) reported that they eat less nutritiously since they began providing care to their spouse and 14 percent report that their appetite has declined (see Table 2). Among the 31 caregivers who reported exercising at least once a week, 33 percent report lower levels of physical activity since they began providing spouse care. Almost one-half of the caregivers who smoke (43 percent) report increasing the number of cigarettes smoked per day since they began providing care for their spouse. Among the 25 caregivers who drink alcohol, only 4 percent report increasing their consumption since providing care to their spouse. Almost one-third of the sample reports taking tranquilizers, anti-depressants, or sedatives to help them sleep, calm them down, or lower anxiety.

Several caregivers reported improvements in their health behavior since providing care to the spouse. Eating more nutritiously, having a better appetite, exercising more frequently, smoking less, and decreasing alcohol consumption were reported by 7 percent, 9 percent, 10 percent, 14 percent, and 28 percent of the caregivers, respectively.

Forty percent of the respondents reported that their physical health had been negatively affected by caring for their spouse. Over half of these caregivers ( 54.5 percent) provided a written explanation of this relationship in the mailed questionnaire. The most commonly mentioned health impact of caregiving included: being tired, exhausted or fatigued $(n=12)$; being under stress $(\mathrm{n}=8)$; sleep disturbances $(\mathrm{n}=5)$; gaining weight $(\mathrm{n}=4)$; high blood pressure $(n=3)$; losing weight $(n=$ 2), limiting opportunities for exercise $(n=2)$, and having difficulty concentrating $(n=2)$. Specific reasons included:

"It just plain wears me out. I never get a full night's sleep."

"I have to be up and about when rest would help with my bronchitis and ear and sinus infections. I am constantly trying to be calm when I'm not."

"He has not been sleeping much the last three weeks so I am not sleeping. I'm stressed out, I've lost 10 pounds, and I'm having a hard time concentrating at work."

"Stress causes my irregular heartbeat."

"Each day is a challenge to deal with my spouse's depression. I'm sure my nervous system is taking a beating. It's tough dealing with a negative attitude. Fortunately, I'm a very positive person most of the time."

"I have gained weight which affects my cholesterol level, which is considered high. I nibble or over eat when I'm upset by his behavior."

"... loss of weight and unable to take daily walks because of not feeling safe in leaving my wife alone."

"It's hard to keep my mind on business because of the lack of sleep and worry."

"I take better care of myself now so I won't be prevented 
Table 3. Caregiver's self reports of spouse's and their own physical health status and chronic illnesses and conditions.

\begin{tabular}{|c|c|c|c|c|}
\hline & \multicolumn{2}{|c|}{$\begin{array}{c}\text { Caregivers } \\
(n=44)\end{array}$} & \multicolumn{2}{|c|}{$\begin{array}{l}\text { Spouses } \\
(n=44)\end{array}$} \\
\hline & $\mathrm{N}$ & $\%$ & $\mathrm{~N}$ & $\%$ \\
\hline \multicolumn{5}{|l|}{ *Self-rated physical health } \\
\hline Poor & 1 & 2.3 & 0 & 0.0 \\
\hline Fair & 15 & 34.1 & 11 & 25.0 \\
\hline Good & 23 & 52.3 & 22 & 50.0 \\
\hline Excellent & 4 & 9.1 & 10 & 22.7 \\
\hline \multicolumn{5}{|l|}{ Chronic illness or condition } \\
\hline Anemia & 2 & 4.5 & 1 & 2.3 \\
\hline Arthritis* & 17 & $38.6^{*}$ & 12 & $27.3^{*}$ \\
\hline Asthma & 1 & 2.3 & 0 & 0.0 \\
\hline Bladder & 6 & 13.6 & 10 & 22.7 \\
\hline Cancer & 4 & 9.1 & 2 & 4.5 \\
\hline Diabetes & 5 & 11.4 & 5 & 11.4 \\
\hline Emphysema* & 3 & $6.8^{*}$ & 0 & $0.0 *$ \\
\hline Gall bladder & 2 & 4.5 & 0 & 0.0 \\
\hline Glaucoma & 6 & 13.6 & 5 & 11.4 \\
\hline Hardening of the arteries & 8 & 18.2 & 6 & 13.6 \\
\hline Heart disease & 10 & $22.7 *$ & 2 & $4.5^{*}$ \\
\hline High blood pressure & 15 & $34.1^{*}$ & 8 & $18.2^{*}$ \\
\hline Kidney disease & 3 & 6.8 & 2 & 4.5 \\
\hline Stroke & 2 & 4.6 & 3 & 6.8 \\
\hline Ulcer (stomach) & 2 & 4.5 & 0 & 0.0 \\
\hline
\end{tabular}

*Difference between caregivers self-reports of their spouse's and of their own physical health status and chronic illnesses and conditions is significant at the .05 level.

from caring for my wife."

One-half of the sample (50 percent) reported that their current health problems limit their activities, and almost half of these caregivers (45.5 percent) provided specific examples of their limitations. The most commonly mentioned responses included: arthritis $(n=5)$, high blood pressure $(n=3)$, and back problems $(n=3)$. Other health problems mentioned by at least one caregiver include: artery bypass, bronchitis, colitis, Crohn's disease, ulcer, kidney trouble, prostate trouble, ear infections, migraines, sinus infections, poor circulation, balance problems, dizzy spells, alcoholism, weight problems, exhaustion, nerves, lack of sleep, sleep disturbances, failing eyesight, difficulty lifting, stress and tension, worry, and depression.

\section{Health behavior coping strategies}

Caregivers reported the frequency of engaging in specific health behaviors to cope with the stress of caregiving in the past month. Exercise, finding comfort in food, spending more time sleeping, taking medications, and drinking alcohol were used as coping strategies by 79.5 percent, 63.6 percent, 52.3 percent, 34.1 percent, and 34.1 percent of the sample, respectively. Exercise, finding comfort in food, taking medications, spending more time sleeping, and drinking alcohol were used on a frequent basis by 20.5 percent, 15.9 percent, 13.6 percent. 4.5 percent, and 2.3 percent respectively.

\section{Comparison of caregivers and spouses physical health}

Caregivers rated their own physical health as significantly poorer than they rate their demented spouse's physical health (see Table 3). Caregivers reported that they had been diagnosed with significantly higher rates of high blood pressure, heart disease, arthritis, and emphysema than their spouses.

\section{Relationship among perceived and objective caregiver burden and physical and mental health variables}

As depicted in Table 4, significant correlations were found between the perceived and objective indicators of burden and physical and mental health outcomes. Specifically, perceived caregiver burden is significantly and positively correlated with on duty hours, caregiving years, sick days, somatization, depression, and worry (all p's < .05). Perceived burden is significantly negatively correlated with self-rated physical health and self-care. The two objective burden indicators, on duty hours and caregiving years, were not significantly related to the physical and mental health variables (with the exception that on duty hours is significantly negatively correlated with self-care; $p<.05)$. Additionally, self-care was significantly and negatively correlated with somatization, depression, and worry. Self-care was significantly 
Table 4. Zero-order correlations, means, and standard deviations of perceived and objective burden and caregiver's physical and mental health variables.

\begin{tabular}{lllllllllll}
1 & 2 & 3 & 4 & 5 & 6 & 7 & 8 & 9 & 10 & 11 \\
\hline
\end{tabular}

\section{Caregiver burden}

1. Perceived burden

2. "On duty" hours

3. Caregiving years ${ }^{b}$

$\begin{array}{rllllllllr}.30 * & .41 * * & -.47 * * * & -.01 & -.44 * * & .25 * & .41 * * & .42 * * & .27 * & -.18 \\ - & .17 & -.16 & -.05 & -.32 * & -.16 & .06 & .18 & .06 & -.13 \\ & - & -.20 & -.06 & -.17 & -.09 & .02 & .13 & -.03 & .09\end{array}$

Caregiver's physical health

4. Self-rated physical heal th ${ }^{\mathrm{a}}$

5. Number of chronic illnesses ${ }^{b}$

6. Self-care ${ }^{\mathrm{a}}$

7. Sick days ${ }^{b}$

8. Somatization ${ }^{\mathrm{c}}$

$$
\begin{array}{ccccccc}
-.54 * * * & .48 * * * & -.26 * & -.53 * * * & -.53 * * * & -.41 * * & .50 * * * \\
- & -.23 & .04 & .32 * * & .21 & .35 * * & -.16 \\
& - & -.17 & -.43 * * * & -.52 * * * & -.24 * & .41 * * \\
& & - & .17 & .29 & .18 & -.24
\end{array}
$$

\section{Caregiver's mental health}

9. Depression ${ }^{\mathrm{d}}$

10. Worry ${ }^{a}$

11. Spirits ${ }^{a}$

\begin{tabular}{lccccccccrrr} 
Mean & 2.7 & 15.8 & 3.3 & 2.7 & 2.2 & 2.2 & 3.8 & 19.0 & 19.8 & 1.8 & 2.6 \\
Standard deviation & 1.2 & 9.2 & 2.3 & 0.7 & 1.9 & 0.7 & 12.3 & 5.2 & 11.9 & 0.8 & 0.7 \\
\hline
\end{tabular}

${ }^{*} \mathrm{p}<.05 ;{ }^{* *} \mathrm{p}<.001$.

${ }^{a}$ One-item indicators, higher scores represent higher levels of the construct (e.g. more burden, better health and self-care, more worry, and better spirits).

bumerical indicators.

${ }^{\mathrm{c}}$ Subscale of the Hopkins Symptom Checklist.

${ }^{\mathrm{d}}$ CES-D score.

and positively correlated with selfrated physical health and spirits.

\section{Discussion}

The purpose of this study was to assess the impact of providing care to a spouse with dementia on caregiver's health behavior and overall physical health. Results suggest that, for some caregivers, providing fulltime care decreased the opportunity and/or incentive to engage in preventive health behaviors (eating nutritiously and exercising) and increased the prevalence of potentially high risk behaviors (overeating and alcohol and substance use).

For example, 40 percent of the caregivers in the present study reported declines in their nutritional status that they associated with the role as care provider. Additionally, over three-fourths of the caregivers reported "finding comfort in food." In general, older adults have been described as particularly vulnerable to health-related consequences of improper nutrition, and this may be particularly true of spouse caregivers. ${ }^{42}$ Caregiving may interfere with proper nutrition in three ways:

- The stress of caregiving may result in poor appetite or overeating, thus directly affecting eating behavior;

- The financial and time demands of providing full-time care may limit the ability, energy and motivation required to purchase and prepare nutritious foods; and

- Because eating behavior is an inherently social activity, ${ }^{43}$ caregivers may perceive few incentives to share meals with a spouse whose cognitive and behavioral declines preclude a mutually satisfying social interaction, ${ }^{44}$ and may take part in fewer social activities that involve meals.

Thus, caregivers may eat alone and use convenience foods more frequently, eat less regularly scheduled 
meals, and reduce the amount and type of food eaten. ${ }^{42}$ Additionally, several study participants reported sizeable weight gains (for one caregiver, over 100 pounds) that resulted in health complications

\section{Problem drinking may result in a pattern of self-neglect and inattention to proper self-care activities ....and interfere with caregiving tasks and responsibilities.}

(increased cholesterol and hypertension) and were attributed to overeating as a response to caregiving stress. Because proper nutrition and optimal body weight contribute to overall physical and mental health, the eating behavior and nutritional status of caregivers is an important area for future research.

Anecdotal evidence suggests that people increase their rate of smoking during times of stress, due in part, to the perception that smoking reduces tension or anxiety.(3) Results from the present study indicate that, for one-half of the smokers, caregivers increase their rate of smoking since providing care to their spouse. The chronic nature of the demands of caregiving may make smoking particularly hazardous for caregivers and their ill spouses due to the direct and passive effects of smoking on morbidity and mortality. ${ }^{45}$ Additionally, the daily demands of caregiving may make quit attempts especially difficult and may trigger a relapse among former smokers. ${ }^{46}$

Only half of the spouse caregivers reported drinking alcohol (56.8 percent). Of this group, over one-fourth report decreasing their alcohol consumption since providing care to their spouse. The restriction that dementia places on social activities may help to explain the decreased intake of alcohol among this sample. Although only one caregiver reported increasing their intake of alcohol since providing care to their spouse, one-third of the sample reported drinking alcohol as a way of coping with the stress of care provision. Although alcohol reduces tension and anxiety when used in moderation, ${ }^{47}$ attention to the potentially dangerous effect of excessive alcohol use among caregivers, especially those who are managing chronic illnesses or conditions, is warranted. An additional concern is that caregiving stress may trigger excessive drinking or a relapse among former problem drinkers. ${ }^{48,49}$ Problem drinking may result in a pattern of self-neglect and inattention to proper self-care activities (nutrition, sleep) ${ }^{49}$ and interfere with caregiving tasks and responsibilities.

One-third of the spouse caregivers reported taking medications (e.g., sedatives, anti-depressants) to cope with the stress of caregiving. As reported elsewhere, caregivers use psychotropic drugs more frequently than the general population, ${ }^{20}$ a comparison group ${ }^{3}$ or matched controls. ${ }^{10,20}$ Although following a prescribed drug regimen may help some caregivers manage stress and anxiety, the potential overuse of such medications and interactions with multiple prescription and non-prescription drugs and nutritional status is a potential threat to the overall health of caregivers.

Although concerns about nutri- tion, eating behavior, smoking, alcohol and medication use among caregivers have been raised, some caregivers reported making improvements in their health behaviors since they began providing care to their spouse, and 80 percent of the sample reported that they view exercise as a means of coping with the stress of caregiving. As discussed by Nowack, ${ }^{29}$ the daily practice of preventive health behaviors may be viewed as a behavioral health coping strategy and as a mediator of the stress-illness relationship. Results from the present study indicate that taking care of one's health was associated with less worry and depression and higher overall spirits. The mechanism by which self-care influences psychosocial outcomes may be an increased sense of personal control over health behaviors, symptoms, or the course of a chronic illness or condition. ${ }^{50}$ Personal control over some life domains (caregiver's own physical health) may be

\section{The mechanism by which self-care influences psychosocial outcomes may be an increased sense of personal control over health behaviors ...}

especially salient for caregivers of dementia patients because the cognitive and behavioral declines associated with the illness cannot be controlled. Responses to open-ended questions in the present study indicate that, for some caregivers, maintaining one's physical health is perceived both as an investment in 
personal well-being and in the ability to provide full-time care at home for as long as possible. This finding may serve as a powerful incentive for other caregivers to engage in preventive health behaviors and decrease high-risk behaviors.

Results also support the view that caregivers are the "hidden patient." ${ }^{51,52}$ First, almost half of the caregivers in the present study report that their overall health status has been negatively affected by providing care for their spouse, particularly in terms of exhaustion, fatigue, and sleep disturbances. The physical health self-ratings reported by the present sample are comparable to those reported in samples of spouse caregivers, ${ }^{17,20}(4)$ but lower than age-based norms. ${ }^{1,35}$ What is notable, however, is that the caregivers in the present study rate their own health as poorer than they rate their ill spouse's health. ${ }^{20}$ Also, caregivers report significantly higher rates of specific illnesses compared to the rates for the spouses for whom they provide care; arthritis, high blood pressure, heart disease, arthritis and emphysema (similar findings were reported elsewhere)..$^{10,17,20}$ Caregivers report that their arthritis and high blood pressure and heart and back problems limit their activities, including their ability to care for their spouse. Because these illnesses and conditions, particularly arthritis, result in dynamic patterns of physical disability and impairments in activities of daily living over time, ${ }^{53}$ the ability to consistently provide full-time care to a spouse with dementia may be compromised. Finally, several of the caregivers mentioned activity limitations due to stress-related illnesses and conditions, including colitis, migraines, and ulcers. ${ }^{54}$ The extent to which these conditions were triggered or aggravated by the demands of caregiving cannot be assessed in the present study, but represent an important focus for future research and caregiver interventions.

\section{The extent to which these conditions were triggered or aggravated by the demands of caregiving ... represent an important focus for future research and caregiver interventions.}

As discussed in the introduction, previous research has documented significant associations between perceived caregiver burden and a variety of mental and physical health outcomes. In this study, perceived burden was positively associated with depression and worry and negatively associated with self-rated physical health and self-care ratings. Objective burden, measured by onduty hours, was negatively correlated with self-care. Additionally, self-care behaviors were negatively associated with depression and positively related to self-rated physical health and spirits. Although the causal relationships among these variables cannot be explored in the present study due to the correlational nature of the data and small sample size, several pathways between burden, self-care, and physical and mental health outcomes can be formulated. Perceived and objective burden may have both indirect and direct effects on self-care activities. Because results indicate that perceived burden is associated with on duty hours, the time available to caregivers for adequate self-care may be limited. Alternatively, perceived burden may be related to selfcare via its indirect effects on depression. Depressed older adults may be less motivated or able to engage in preventive health behaviors, manage their chronic illnesses and conditions, or to provide care for their spouse. ${ }^{33,34}$ In other words, the impact of perceived burden on selfcare may be mediated through depression (especially given the magnitude of the correlation between depression and self-care, $\mathrm{r}=-.52$ ). Testing the potential mechanisms of the relationship between burden, self-care and physical and mental health outcomes is a fruitful area of future research.

Some of the limitations of the present study should be noted. Although the sample size is relatively small, all participants were recruited from a statewide list of patients who sought a consultation or treatment from a cognitive disorders clinic at a large university. This recruitment strategy may be less likely to result in a particularly distressed group than one derived from support group membership lists, although the sample cannot be assumed to be representative of all spouse caregivers in the community. Due to the small sample size, gender differences were not explored. Because previous research has documented both greater impact of stress on health ${ }^{55,56}$ and alcohol consumption ${ }^{57}$ among women than men, additional research is needed to clarify the potential gender differences in the impact of caregiving on health behaviors. ${ }^{17,58}$ All data collected for the present study is self reported. As discussed recently, ${ }^{4}$ self-reports of physical 
health status may be biased by the depression and anxiety that caregivers may experience.

A comparison group of caregivers of non-demented spouses was not available. Thus, it is not possible to consider whether the findings are unique to dementia caregivers or also pertain to other types of spouse caregivers. Finally, the data used in the present study is cross-sectional; therefore, the stability of the relationships cannot be assessed. As mentioned previously, however, caregivers from the present study also completed daily health diaries for 10 weeks to permit the examination of patterns of change over time in the perceived stress of caregiving, health behaviors, and mental and physical health status. Preliminary results of this data indicated high levels of variability among caregivers, but a strong pattern of covariation over time in perceived stress and caregiver health. ${ }^{30}$ This relationship was mediated, for several caregivers, by the specific type of caregiving stress experienced (hassles with behavior problems, cognitive changes, or activities of daily living). ${ }^{31}$

\section{Conclusion}

Data from the present study indicate that caregivers report several high risk health behaviors and the tendency to engage in these behaviors as a response to the stress of caregiving. Although additional research based on large representative samples of community-dwelling caregivers is needed, results suggest that interventions that focus on the physical health and health behaviors of caregivers is warranted. Because older adults may be more willing to seek help for their physical health concerns than for symptoms of men- tal distress, ${ }^{59}$ interventions that emphasize health behavior change may be attractive to a wide range of caregivers. The prevention of health problems and the self-management of existing illnesses and conditions may extend caregiver's ability to provide full-time in-home care, reduce the likelihood of the demented patient being institutionalized, and promote positive physical and psychosocial outcomes among caregivers. Because increasing numbers of older adults will provide care for a spouse with a dementia in the future, the development of interventions with a preventive focus is especially timely. $\square$

\section{Footnotes}

(1)Exceptions to this focus on self reports include: a) ongoing research by GallagherThompson et $\mathrm{al}^{14}$ that examines the impact of caregiving stress on cardiovascular risk, b) completed work by Kiecolt-Glaser et al. ${ }^{60}$ that suggests that caregiving stress compromises immune function, and c) completed work by Moritz et al. ${ }^{17}$ in which living with a cognitively impaired spouse was found to increase systolic blood pressure in men not treated for hypertension.

(2) Achecklist was provided for this purpose and included: diabetes; high blood pressure/hypertension; heart trouble; circulation problems/hardening of the arteries; paralysis; effects of stoke; arthritis/ rheumatism; stomach ulcer; asthma; glaucoma/pressure behind the eye; cataracts; cancer; liver trouble/jaundice; gall bladder trouble; kidney trouble; bladder trouble; broken hip; broken bones, anemia; Parkinson's disease; and emphysema.

(3) Empirical evidence suggests a strong relationship between perceived stress and changes in smoking status, although the precise causal mechanism of this relationship has not been isolated. ${ }^{61}$ Specifically, it is difficult to determine whether stress inhibits quit attempts and/or increases smoking rates or if attempts to quit fail because of perceived stress.

(4) In the present study, self-rated physical health was rated as poor, fair, good, and excellent by 4.5 percent, 31.8 percent, 52.3 percent, and 9.1 percent of the sample, respectively; these ratings are very comparable to the 5.1 percent, 26.5 percent, 53.1 percent, and 15.3 percent for the Pruchno et al. ${ }^{13}$ sample of 315 spouse caregivers. Similarly, Moritz et al. ${ }^{17}$ reported that among her sample of 318 spouse pairs, 39.2 percent and 59.6 percent rated their health as poor/fair and good/excellent.

\section{Acknowledgments}

This research was supported by NIA grant I P50 AG08671-03 to the Michigan Alzheimer's Disease Research Center, S. Gilman, MD, Program Director, C. M Connell, PhD, Director, Education and Information TransferCore; and by the Office of the Vice President for Research at the University of Michigan, C.M Connell, Principal Investigator. The author gratefully acknowledges J. Schulenberg and M. Gallant for their helpful critique of an earlier version of the manuscript; M. Ryan for her assistance with manuscript preparation; and each of the volunteer subjects for their participation.

\section{References}

1. Stone R, Cafferata GL, Sangl J: Caregivers of the frail elderly: A national profile. Gerontologist, 1987;27:616-626

2. Horowitz A: Family caregiving to the frail elderly. In: Eisdorfer C (ed.): Annual review of gerontology and geriatrics. New York, Springer Publishing Co., 1985;5:194-246

3. George L, Gwyther L: Caregiver well-being: A multidimensional examination of family caregivers of demented adults. Gerontologist, 1986;26:253-259

4. Schultz R, Visintainer P, Williamson GM: Psychiatric and physical morbidity effects of caregiving. J Gerontol, 1992;45:P181-191

5. Zarit SH, Reever KE, Bach-Peterson J: Relatives of the impaired elderly: Correlates of feelings of burden. Gerontologist, 1980;20:649-655

6. Cantor MH: Strain among caregivers: A study of experience in the United States. Gerontologist, 1983;23:597-604

7. Montgomery RJV, Gonyea JG, Hooyman NR: Caregiving and the experience of subjective and objective burden. Fam Relat, 1985;34:19-26

8. Chenoweth B, Spencer B: Dementia: The experience of family caregivers. Gerontologist, 1986;26:267-272

9. Snyder B, Keefe K: The unmet needs of family caregivers for frail and disabled adults. Soc Work Heal th Care, 1985;10:1-14 10. Haley WE, Levine EG, Brown SL, Berry JW, Hughes GH: Psychological, social, and health consequences of caring for a relative with senile dementia. J Am Geriatr Soc, 1987;35:405-411

11. Baumgarten M: The health of persons giving care to the demented elderly: A critical review of the literature. J Clin Epidemiol, 1989;42:1137-1148

12. Moritz DJ, Kasl SV, Berkman LF: The 
health impact of living with a cognitively impaired elderly spouse: Depressive symptoms and social functioning. J Gerontol, 1989;44:517-527

13. Pruchno RA, Kleban MH, Michaels JE, Dempsey NP: Mental and physical heal th of caregiving spouses: Development of a causal model. J Gerontol, 1990;45:P192199

14. Gallagher-Thompson D, Walsh W, Lovett S, Koin D: The relationship among indices of physical and psychological distress in family caregivers. Gerontologist, 1991;31:90A

15. Koin D, Rinki M, Walsh WA, GallagherThompson LD: Cardiovascular risk factors in caregivers of patients with progressive dementia. Gerontologist, 1991;31:90A

16. Walsh WA, Yoash-Gantz, Rinki M, Koin D, Gallagher-Thompson D: The use of alcohol, exercise, smoking and psychotropic drugs among female caregivers. Gerontologist, 1991;31:90A

17. Moritz DJ, Kasl SV, Ostfeld AM: The health impact of living with a cognitively impaired elderly spouse: Blood pressure, self-rated health, and health behaviors. J Aging Health, 1992;4:244-267

18. Pratt CC, Schmall VL, Wright S, Cleland M: Burden and coping strategies of caregivers to Alzheimer's patients. Fam Relat, 1985;34:27-33

19. Colerick EJ, George LK: Predictors of institutionalization among caregivers of patients with Alzheimer's disease. J Am Geriatr Soc, 1986;34:493-498

20. Pruchno RA, Potashnik SL: Caregiving spouses physical and mental health in perspective. J Am Geriatr Soc, 1989;37:697-705

21. Aronson MK: Patients and families: Impact and long-term management implications. In: Aronson MK (ed.): Understanding Alzheimer's disease, New York, NY, Charles Scribner and Sons, 1988:147-163

22. Cohen D: Issues in psychological diagnosis and management of the cognitively impaired aged. In: Eisdorfer C, Fann E (eds.): Treatment of psychopathology in aging. New York, Springer, 1982:192-211

23. Barusch AS: Problems and coping strategies of elderly spouse caregivers. Gerontologist, 1988;28:677-685

24. Mace NL, Rabins PV: The 36-hour day. Baltimore, MD, John Hopkins University Press, 1981

25. Biegel DE, Sales E, Schulz R: Caregiving in Alzheimer's disease. In: Biegel DE, Sales E, Schulz R (eds.): Family caregiving in chronic illness. Newbury Park, CA, Sage Publications, inc., 1991:147-163

26. Belloc NB, Breslow L: Relationship of physical health status and health practices. Prev Med, 1972;1:409-421

27. United States Department of Health, Education and Welfare: Obesity and health:
A source book of current information for public health personnel. Public Health Service, Division of Chronic Diseases, Publication No. 1485. Washington, DC, U.S. Government Printing Office

28. Berkman LF, Breslow L: Health and ways of living: The Alameda County Study. New York, Oxford University Press, 1983 29. Nowack KM: Coping style, cognitive hardiness, and health status. J Behav Med, 1989;12:145-158

30. Connell CM, Schulenberg J: Daily variation in the physical and mental health impact of caregiving. Gerontologist, 1990;30:236A

31. Connell CM, Gallant MP, Schulenberg J: Daily variation in caregiving hassles: A factor analytic approach. Gerontologist, 1991; 31:193A

32. Frerichs RR, Aneshensel CS, Yokopenic PA, Clark VA: Physical health and depression: An epidemiologic survey. Prev Med, 1982;11:639-646

33. Anehensel CS, Frerichs RP, Huba GJ: Depression and physical illness: A multiwave, nonrecursive causal model. J Health Soc Behav, 1984;25:350-371

34. Gallant MP, Connell CM: Daily physical and mental health self-ratings and their relationship with depression. Under review, 1993 35. National Center for Health Statistics: Current estimates from the National Health Interview Survey, United States, Series 10, Number 164, 1986

36. LaRue A, Bank B, Jarvik L, Ketland M: Health in old age: How do physicians' ratings and self-ratings comapare? J Gerontol, 1979;34:687-691

37. Mossey JM, Shapiro E: Self-rated health: A predictor of mortality among the elderly. AJPH, 1982;72:800-808

38. Lawton MP, Kleban MH, Moss M, Rovine M, Glocksman A: Measuring caregiving appraisal. J Gerontol, 1989;44: 61-70

39. Derogatis LR, Lipman RS, Rickels K, Uhlenhuth EH, Covi L: The Hopkins Symptom Checklist (HSCL): Self-report symptom inventory. Behav Sci, 1974;19:1-15

40. Radloff L: The CES-D Scale: A self-report depression scale for research in the general population. Appl Psychol Measurement, 1977;1:385-401

41. Devins GM, Orme CM: Center for Epidemiologic Studies Depression Scale. In: Keyser DJ, Sweetland RC (eds.): Test critiques. Kansas City, Missouri, 1985

42. McIntosh .WA, Shifflett PA, Picou JS: Social support, stressful events, strain, dietary intake, and the elderly. Med Care, 1989; 27:140-153

43. Fieldhouse P: Food and nutrition: Customs and culture. London, Croom Helm, 1986 44. Umberson D: Family status and health behaviors: Social control as a dimension of social integration. J Health Soc Behav, 1987;
28:306-319

45. United States Preventive Services Task Force: Guide to clinical preventive services: An assessment of the effectiveness of 169 interventions. Baltimore, MD, Williams \& Wilkins, 1989

46. Marlatt GA, Gordon JR: Relapse prevention: Maintenance strategies in the treatment of addictive behaviors. New York, NY, Guilford Press, 1985

47. Stockwell T: Stress and alcohol. Stress Med, 1985;1:209-215

48. Rosenberg H: Relapsed versus non-relapsed alcohol abusers: Coping skills, life events, and social support. Addictive Behav, 1983;8:183-186

49. Finney JW, Moos RH: Life stressors and problem drinking among older adults. In: Galanter RC (ed.): Alcoholism, New York: Plenum Press, 1984.

50. Affleck G, Tennen H, Pfeiffer C, Fifield $\mathrm{J}$ : Appraisals of control and predictability in adapting to a chronic disease. J Pers Soc Psychol, 1987;53:273-279

51. Fengler AP, Goodrich N: Wives of elderly disabled men: The hidden patients. Gerontologist, 1979;19:175-183

52. Zarit SH, Ory N, Zarit J: The hidden victims of Alzheimer's disease: Families under stress. New York, NY, New York University, 1985

53. Verbrugge LM: Disability transitions for older persons with arthritis. J Aging Health, 1992;4:212-243

54. Taylor SE: Stress and the development of specific illnesses. In: Taylor SE (ed.): Health Psychology, New York, Random House, 1986:176-200

55. Pearlin LI, Schooler C: The structure of coping. J Health Soc Behav, 1978;19:2-21

56. Kessler RC: A strategy for studying differential vulnerability to the psychological consequences of stress. J Health Soc Behav, 1979;20:100-108

57. Cronkite RC, Moos RH: The role of predisposing and moderating factors in the stress-illness relationship. J Health Soc Behav, 1984;25:372-393

58. Pruchno RA, Resch NL: Husbands and wives as caregivers: Antecedents of depression and burden. Gerontologist, 1989;29: 159-165

59. Houpt JL, Orleans CS, George LK, Brodie HKH: The role of psychiatric and behavioral factors in the practice of medicine. Am J Psychiatry, 1980;137:37-47

60 Kiecolt-Glaser JK, Glaser R, Shuttleworth EE, Dyer CS, Ogrocki P, Speicher CE: Chronic stress and immunity in family caregivers of Alzheimer's disease patients. Psychosom Med, 1987;49:523-535

61. Cohen S, Lichtenstein E: Perceived stress, quitting smoking, and smoking relapse. Health Psychol, 1990;9:466-478 